\title{
Tagliare è un verbo da coniugare, non solo da imparare
}

\author{
di Elio Borgonovi
}

Le politiche riguardanti i sistemi di tutela della salute, non solo di quelli che adottano il modello di SSN ma anche di quelli che adottano il modello fondato su mutue o casse o su assicurazioni private, negli ultimi anni hanno posto l'accento soprattutto sul verbo tagliare, di volta in volta ammorbidito con i verbi riorganizzare, razionalizzare, riformare. Tagliare la spesa, o il tasso di sviluppo della spesa, poiché i sistemi di welfare, più o meno esteso, dei Paesi sviluppati non sarebbero più sostenibili. Si è sentito spesso sottolineare che il problema per l'Europa può essere condensato in tre cifre: il 7\% della popolazione mondiale, il $25 \%$ del reddito totale prodotto, il 50\% della spesa globale per welfare.

Si dà per scontato che il riequilibrio di queste cifre debba passare attraverso la riduzione delle spesa per welfare dei Paesi europei senza prendere nemmeno in considerazione la soluzione alternativa secondo la quale il riequilibrio tra reddito prodotto e spesa potrebbe avvenire aumentando la quota di spesa nei Paesi in sviluppo, o ex rapido sviluppo viste le difficoltà degli ultimi due anni dei BRICS, o dei Paesi che stanno cercando faticosamente una propria via di sviluppo in un mondo che appare colpito dal virus del basso tasso di crescita. Le proposte per il rilancio della crescita finora discusse nei molti vertici economici che si sono susseguiti a ritmo sempre più incalzante, ma che per ora si sono tradotte in dichiarazioni, auspici, accordi molto generici possono essere riassunte nei seguenti termini: la richiesta di aumentare i consumi nei Paesi in surplus commerciale (esempio Germania), la transizione dell'economia cinese da un modello trainato dalla spesa per investimenti pubblici a un modello trainato dall'aumento dei consumi privati, lo stimolo del quantitative easing alle banche per sostenere finanziamenti all'economia reale consentito dai tassi di interesse vicini allo zero o addirittura negativi. Scarsa attenzione è stata dedicata alla riflessione sul fatto che la crescita economica dell'ultima metà del ventesimo secolo è stata trainata soprattutto dal consolidamento della classe media (solo dagli anni Novanta si è avuta un'inversione di tendenza) e dall'aumento della spesa pubblica e privata per il welfare, ossia il miglioramento della qualità di vita. Partendo da questa considerazione si può ritenere che "tagliare" sia un verbo da "coniugare" e non solo da "imparare", come sostengono coloro che teorizzano la fine del periodo di vacche grasse e che per realizzare il miglioramento sia necessario "affamare la bestia" (lo starve the beast come dicono gli inglesi).

La prima declinazione riguarda concetti che sembrano precisi ma che in effetti sono generali e generici. Infatti quasi sempre si evoca, nel dibattito scientifico o di fronte all'opinione pubblica, nei documenti di programmazione o di indirizzo, nelle leggi, nei decreti, la necessità di tagliare i costi, gli sprechi, le inefficienze. Non di rado gli obblighi formali a intervenire su questi ambiti sono sostenuti dalla documentazione di casi o di situazioni che evidenziano sprechi, inefficienze o differenze anomale dei prezzi d'acquisto. Si citano spesso e in modo ricorrente i casi del prezzo delle siringhe, dei fili di sutura per uso chirurgico, dei guanti dei medici e degli infermieri e di tanti altri beni di consumo che hanno variazioni da 1 a 4, 1 a 51 a n volte in diverse aziende ospedaliere o territoriali. Si possono aggiungere anche i frequenti riferimenti agli sprechi derivanti da apparecchiature, attrezzature acquistate e mai utilizzate o utilizzate per un limitato numero di ore. A riguardo si propongono le seguenti riflessioni: 
- a volte i confronti dei prezzi dei beni appena citati e di altri sono di tipo giornalistico perché fanno notizia (quante volte i ricercatori sono stati contattati da giornalisti che chiedevano dati e risultati "notiziabili"), ma non sempre sono attendibili perché di fatto confrontano cose diverse pur nell'ambito di una stessa tipologia o classe merceologica;

- anche quando sono attendibili e quindi mettono in evidenza sprechi e inefficienze reali, riguardano non di rado livelli di spesa non elevati rispetto al totale: correttamente si può dire che sprechi e inefficienze vanno comunque combattuti e tagliati anche se di dimensioni non elevate, ma non si può negare che l'effetto mediatico di queste informazioni e notizie è spesso esagerato rispetto al problema reale;

- anche quando poi si tratta di sprechi e inefficienze reali e che incidono in modo rilevante sul totale della spesa di una ASL, di una azienda ospedaliera, di una regione, dell'intero sistema (dipende dal livello di riferimento delle analisi), si compie l'errore di ritenere che tagliare gli sprechi e le inefficienze sia semplice in quanto si afferma che "basta la volontà", "basta applicare i sani principi del management", "basta fare quello che hanno fatto le imprese private".

Ci si dimentica che anche nel mondo delle imprese tagliare gli sprechi e le inefficienze non sempre è facile tanto è vero che molte imprese falliscono o perdono rilevanti quote di mercato e sono costrette a ridimensionarsi. Ci si dimentica che non è facile passare dall'analisi, dalla individuazione del problema, alla sua soluzione. Utilizzando un paragone di tipo sanitario si potrebbe dire che non sempre è facile passare dai sintomi, alla loro interpretazione, alla diagnosi (individuazione della malattia), ma è ancor più difficile passare dalla diagnosi alla terapia e alla sua applicazione efficace. Coloro che di fronte alla individuazione di tipologie di sprechi e inefficienze propongono di applicare gli interventi positivi attuati in alcuni ospedali, aziende sanitarie, regioni definite "virtuose" agiscono come coloro che, dopo aver effettuato una certa diagnosi su un paziente, propongono per lo stesso terapie suggerite ad altri pazienti. Se si tratta di patologie comuni e di utilizzo di farmaci o di terapie aventi un valore generale, la cosa può funzionare, ma nel caso di sprechi e inefficienze le cause sono sempre diverse perché sono diverse le aziende e i contesti in cui essi sono generati.

Un secondo ambito nel quale va coniugato il verbo "tagliare" riguarda la domanda superflua e inappropriata. $\grave{E}$ appena il caso di ricordare che nel campo della tutela della salute vale ancora il "circolo vizioso" della offerta che genera la propria domanda. In altre parole, il medico o altro professionista della salute, la struttura di offerta hanno ancora una influenza rilevante nei confronti del paziente che di fronte a un problema di salute si trova in molti casi in uno status emotivo di sudditanza. A questo classico circolo vizioso se ne possono aggiungere altri due. Il primo riguarda la richiesta del "secondo parere" che viene previsto dalle normative più avanzate a tutela del paziente o che comunque molto spesso viene richiesto soprattutto nei casi di diagnosi di gravi malattie a cui si può aggiungere un terzo o un quarto parere. Questo comportamento è ancor più accentuato nell'ambito del servizio pubblico di fronte a prestazioni gratuite o con partecipazione (ticket) di limitata entità, ma è anche presente quando il paziente deve pagare direttamente la prestazione (spesa privata out of pocket) o può far ricorso a fondi, casse, assicurazioni private. Un secondo fattore che rende difficile "tagliare la domanda di servizi" è determinato dall'accesso alla rete. Ciò ha reso possibile comportamenti di auto-diagnosi e auto-cura che inducono la domanda e rendono più difficile "tagliare" richieste improprie. Diventa sempre più difficile per i medici resistere alle richieste di prestazioni derivanti da questo canale informativo, molte volte distorto, e non ci si può richiamare alla loro deontologia professionale e senso etico. La terza coniugazione del verbo "tagliare" riguarda le prestazioni e i servizi inappropriati. Si tratta del problema precedente analizzato però dal lato dell'offerta. Si afferma che è necessario e possibile tagliare semplicemente in modo agevole tramite la "razionalizzazione" sulla base di conoscenze scientifiche e di evidenze di efficacia. Nessuno può contestare il richiamo a questi principi, ma vanno ricordate alcune caratteristiche del sistema di tutela della salute che possano indebolirne la concreta applicazione. Innanzitutto il fatto che le problematiche di salute sono influenzate anche da aspetti emotivi e non possono considerare solo fattori di razionalità scientifica. Non a caso l'effetto placebo è stato dimostrato con il metodo scientifico del doppio cieco e quindi si può ritenere che il medico in scienza e coscien- 
za possa erogare prestazioni o servizi che contribuiscono al benessere percepito dal paziente anche se in astratto potrebbero essere considerati inappropriati o superflui. Va poi ricordato che esiste una grande differenza tra evidenza scientifica prodotta in laboratorio e nella "sperimentazione clinica controllata" e concreta applicazione di terapie su una popolazione generale in contesti assai diversi tra loro. Trattamenti considerati inappropriati rispetto ai parametri individuati in laboratorio o nelle sperimentazioni cliniche, potrebbero non essere considerati tali quando applicati a pazienti "reali" che reagiscono in modo diverso da quelli sottoposti a sperimentazione clinica. Inoltre, chi opera all' interno del sistema sa benissimo che diversi medici o diverse strutture, anche di elevata qualificazione e specializzazione, affrontano in modo diverso le stesse problematiche di salute. Anche medici o équipe che fanno parte delle stessa "scuola" affrontano in modo diverso, in misura più o meno ampia, le stesse problematiche. Ciò che appare inappropriato a qualcuno è invece considerato appropriato da altri. Infine esiste il fenomeno della "medicina difensiva" rispetto al quale sono inutili o inefficaci proclami. Esiste il paradosso solo apparente in rapporto al quale la maggiore tutela del paziente può causare un aumento della medicina difensiva ossia di prestazioni dettate non da una reale esigenza di salute, ma dalle esigenze di precauzione dei medici, infermieri e altri operatori rispetto a eventuali contestazioni o cause legali da parte di pazienti. Non si vuol certo negare l'esistenza di un elevato livello di inappropriatezza agendo sulla quale sarebbe possibile tagliare la spesa senza ridurre i livelli di salute. Si vuol solo attirare l'attenzione sul fatto che l'inappropriatezza è una conseguenza, usando l'analogia medica è un sintomo, ma che per eliminarla occorre agire sulle cause che sono complesse e spesso tra loro interdipendenti. In un certo senso l'inappropriatezza è diventata una "patologia cronica" dei sistemi di tutela della salute, anzi ci si trova di fronte a una multicronicità data la numerosità delle cause, che non può essere affrontata con gli strumenti con i quali si affrontano "patologie acute" dovute a cause specifiche e ben delimitate quali possono essere forme di sprechi e inefficienze dovute ad acquisti a prezzi più elevati, acquisti di una attrezzature non utilizzate, bassi livelli di produttività dovuti a cattiva organizzazione dei processi assistenziali ecc.

La quarta coniugazione del verbo tagliare riguarda non tanto le prestazioni e i servizi quanto le strutture di offerta con l'obiettivo di ridurre $i$ costi fissi. Interventi di riduzione e aggregazione di unità organizzative che non raggiungono dimensioni di operatività idonee a garantire la sicurezza dei pazienti, e perfino la chiusura o la trasformazione di ospedali di piccole dimensioni, sembrano essere diventati un po' più semplici anche nel nostro Paese dopo l'applicazione del decreto sugli standard strutturali, funzionali, operativi. Questa norma ha dato più forza alle regioni nell'attuare interventi di razionalizzazione della struttura di offerta che consenta di ridurre i costi fissi di struttura. L'orientamento verso la deospedalizzazione consentita dai progressi della scienza, il potenziamento dell'assistenza in strutture territoriali o a domicilio, l'adozione di modelli di organizzazione a rete hanno reso più incisivi gli interventi delle regioni che sono state meno condizionate dalla difesa corporativa di "posizioni" formali di responsabilità (i vari capi e capetti intermedi). Anche la riduzione della degenza media e la più agevole mobilità hanno in un certo senso contribuito a ridurre le resistenze della popolazione rispetto a riduzioni di questo tipo, soprattutto se esse sono state bilanciate con il potenziamento di servizi comunque vicini ai pazienti, esempio prelievi, controlli, visite in strutture diurne diffuse sul territorio.

Dalle considerazioni svolte deriva un messaggio chiaro: l'elemento critico non è l'individuazione di ciò che dev'essere tagliato, l'identificazione di cosa fare ma l'individuazione di come fare, degli strumenti per attuare gli indirizzi e gli interventi. A riguardo si può dire che lo strumento più semplice e che può apparire più efficace è quello di creare vincoli esterni, nel caso del SSN il vincolo del Fondo Sanitario Nazionale.

Si ritiene possibile perseguire l'obiettivo di tagli dei costi, sprechi, inefficienze, inappropriatezze sottoponendo le regioni e a cascata le aziende sanitarie locali e le aziende ospedaliere al duplice vincolo esterno dell'obbligo di garantire $i$ $L E A$ e di realizzare un equilibrio di bilancio. Ma come in una pentola a pressione, spesso accade che in presenza di vari vincoli il sistema trovi uno sfogo nei punti più deboli. Durante gli anni Ottanta e Novanta il punto debole era costituito dal vincolo del finanziamento per cui il sistema trovava un equilibro tramite disavanzi rilevati a consuntivo (sfondamento dei tetti di spesa) coperti, seppur con ritardo, con interventi di ripiano. A partire dal nuovo Secolo e in misura sempre più forte a seguito della normativa sui piani di rientro e su tutte le regioni con i provvedimenti urgenti 
di finanza dal 2011, il punto debole è diventato quello dei livelli di assistenza effettivamente garantiti. I LEA non solo sono rimasti fermi al 2001 e non sono stati aggiornati salvo modifiche e integrazioni parziali e limitate, ma sono stati garantiti in concreto in termini differenziati e progressivamente divaricati tra le regioni. Vi sono stati indubbiamente alcuni effetti positivi in termini di taglio dei costi, delle strutture inutili o addirittura pericolose, di sprechi, inefficienze, inappropriatezze ma per molte aziende sanitarie, aziende ospedaliere, vi è stato il rispetto dei vincoli tramite un allungamento delle liste di attesa, la posticipazione di prestazioni e servizi all'anno successivo, la riduzione della qualità delle cure dovuta a carenza di personale. Non è questa la sede per fare un confronto tra effetti postivi ed effetti negati$v i$, ma è doveroso richiamare l'attenzione sull'esigenza di attuare un monitoraggio più attento di questi effetti da parte di organismi terzi e indipendenti rispetto ai "soggetti" direttamente coinvolti nelle politiche (governo, MEF, Ministero della salute, regioni, aziende sanitarie locali e aziende ospedaliere, provider privati accreditati del Sistema Sanitario Nazionale).

$\grave{E}$ opportuno e doveroso richiamare l'attenzione sul fatto che il verbo tagliare non può essere coniugato indipendentemente dai tempi per esempio tagliare oggi spese per la prevenzione, per il rinnovo delle tecnologie sanitarie, per la ricerca e l'innovazione può causare (e in genere causa) un aumento superiore di costi, sprechi, inefficienze, inappropriatezze, costi futuri. Per evitare questo effetto non desiderabile, si può sottolineare che è possibile "tagliare" in modo efficace e tenendo conto delle specifiche realtà tramite l'introduzione di sistemi di contabilità dei costi, di analisi costibenefici, di analisi dei rendimenti, analisi make or buy, in generale sistemi di controllo manageriali, o di programmazione e controllo interno. In questo modo è possibile attuare una reale spending review. Tuttavia questi interventi sono fattibili solo se all'interno delle strutture che erogano $i$ servizi sono presenti o sono sviluppate tramite la formazione continua, professionalità in grado di utilizzare questi strumenti in modo corretto. Non di rado accade invece che essi siano introdotti ma non siano utilizzati per le decisioni, o siano addirittura utilizzati in modo distorto, e a volte "perverso" a fini di potere organizzativo, da persone che conoscono le tecniche ma non la logica di questi sistemi di controllo manageriale o da persone che hanno obiettivi individuali diversi da quelli della funzionalità ed economicità della gestione.

L'effetto congiunto di vincoli esterni e adozione di strumenti conoscitivi interni finalizzati all'individuazione di specifici sprechi e inefficienze può essere positivo, anche in modo esponenziale, se si usa la leva della responsabilizzazione. Il modo migliore per coniugare il verbo tagliare è la responsabilizzazione dei pazienti per incentivarli a richiedere solo prestazioni necessarie ed evitare accertamenti e prestazioni inutili, la responsabilizzazione dei medici e degli altri professionisti non solo con riguardo alla qualità dei loro atti di assistenza ma anche sul piano organizzativo e del contenimento dei costi, la responsabilizzazione dei dirigenti riconoscendo però a essi una adeguata autonomia di scelta, la responsabilizzazione dei policy maker con riguardo alla valutazione ex ante ed ex post dell'impatto delle politiche. Si può dire che in un sistema complesso i principi, gli indirizzi, i criteri posti dall'alto (esempio Stato) o da vincoli esterni, producono effetti complessivi positivi solo se esistono migliaia e centinaia di migliaia di persone "responsabili" e "responsabilizzate". Con limitato rischio di essere contraddetto, mi sento di affermare che negli ultimi anni e non solo in Italia è stata imboccata una via molto discutibile per cercare di recuperare un equilibrio di sistemi economici e di tutela della salute in gravi difficoltà: quella del rafforzamento di vincoli esterni, delle soluzioni standardizzate, delle procedure e dei controlli rigidi, della riduzione dei margini di autonomia $i$ cui effetti sono la deresponsabilizzazione, l'esatto contrario di ciò che servirebbe. Persone deresponsabilizzate privilegiano l'esigenza di "mettersi a posto" con la legge, con gli standard, con le procedure, con i rischi di essere chiamati in giudizio per presunta mala sanità rispetto alla esigenza di garantire la migliore qualità dell'assistenza. Per fortuna dei sistemi complessi e in particolare di quelli pubblici, esistono ancora nel SSN tantissime persone che sono qualificate dalle teorie sulla Public Service Motivation come giver (ossia persone che perseguono l'interesse generale, nel caso specifico dei pazienti, indipendentemente dagli incentivi espliciti). Il SSN italiano può ancora essere considerato di buona qualità, pur con le grandi e crescenti differenze tra regioni, perché il numero dei giver è superiore a quello dei taker (coloro che si attendono ricompense individuali superiori ai contributi che essi danno al perseguimento dell' interesse generale). Per altro è improprio parlare di fortuna ma è più corretto parlare di una componente essenziale della resilienza (capacità di 
reagire agli shock determinati da vincoli e da incongruenze esterne) del SSN: la componente della professionalità, della motivazione intrinseca, della deontologia, dei valori etici presente in tantissime persone.

Dopo aver posto l'attenzione sul come tagliare si può affrontare un altro tema, quello di identificare chi deve tagliare. A livello di ricerca e di dibattiti spesso accade un fenomeno strano. Coloro che si limitano a individuare e analizzare e denunciare gli sprechi, le inefficienze, i costi eccessivi, l'inappropriatezza si autorappresentano nella categoria di chi vuol tagliare $i$ costi inserendo coloro che evidenziano le difficoltà automaticamente nella categoria di chi resiste al cambiamento, alla razionalizzazione, all'innovazione. Non è il caso di citare il detto popolare "can che abbia non morde" ma nessuno può contestare il fatto è ben più facile osservare i sintomi (per proseguire nelle analogie mediche) che non definire una terapia adeguata e applicarla quando ci si trova di fronte a comportamenti consolidati da anni dietro ai quali stanno privilegi o interessi economici più o meno leciti. Chi opera sul campo e chi si dedica alla ricerca applicata agli interventi organizzativi si trova in una situazione complessa nella quale deve riuscire a trovare un equilibrio tra "cosa si dovrebbe o si potrebbe fare" e "cosa concretamente si riesce a fare" con le risorse disponibili (sempre più scarse), con i vincoli normativi che spesso pongono pastoie burocratiche, con ospedali o strutture edilizie obsolete, apparecchiature e attrezzature diagnostiche e di cura inadeguate (che non è possibile cambiare per mancanza di risorse per investimenti), con personale che negli ultimi anni è stato ridotto numericamente dai vincoli del turnover, con i tagli della formazione. Chi amministra e chi opera si trova spesso tra l'incudine (dei bisogni crescenti, della pressione dell'opinione pubblica, degli obblighi assistenziali imposti da leggi) e il martello (degli organi di controllo, delle denunce per mala sanità, dei media che spesso cercano lo scoop più che la verità). Purtroppo non sempre il martello batte dalla pare giusta ossia colpisce gli sprechi, le inefficienze, le inappropriatezze reali ma a volte (e non raramente) batte dalla parte sbagliata ossia colpisce coloro che operando per il miglioramento del sistema e a favore dei pazienti e della buona salute toccano interessi illeciti e malavitosi (che ovviamente sono i più reattivi e pericolosi). In conclusione alcune brevissime considerazioni. Per indurre le persone a tagliare nel modo giusto si può agire su due differenti leve: agire su obblighi, minacce di penalizzazioni, incentivi monetari o di carriera o, viceversa, privilegiare l'azione su motivazioni intrinseche, autonomia gestionale e responsabilizzazione sui risultati, promozione di sistemi di controllo interno. Per chi scrive il secondo approccio deve ritenersi più efficace anche in una prospettiva di mediolungo termine, mentre da almeno un decennio (e forse anche prima) è stato privilegiato il primo approccio, la cui efficacia non di rado si è rivelata simile a quella delle grida manzoniane o ha prodotto l'effetto di "buttar via il bambino (servizi di qualità) con l'acqua sporca (inappropriatezze, sprechi, inefficienze, corruzioni)". Si può poi dire che è più facile tagliare $i$ costi degli altri rispetto ai propri, per esempio quelli delle regioni o degli enti locali rispetto allo Stato, quelli dell'azienda sanitaria locale o dell'ospedale vicino piuttosto che il proprio per cui a molti fautori dei tagli si potrebbe dire "medico cura te stesso". È più facile dare consigli (come fanno spesso i ricercatori, gli accademici, $i$ consulenti, ifrequentatori di talkshow più o meno seri e documentati) piuttosto che impegnarsi in prima persona nel tagliare nel modo giusto assumendosi le responsabilità dirette. È difficile e richiede tempo aggregare tutti coloro che avrebbero interesse ai tagli, per esempio perché si liberano risorse per impieghi più efficaci per avere una maggiore forza nel combattere coloro che hanno interesse a non tagliare, poiché questi ultimi capiscono subito le conseguenze negative delle razionalizzazioni per i propri interessi.

Bisogna conoscere a fondo il funzionamento dei sistemi complessi, comprendere le difficoltà dei processi di cambiamento, avere la pazienza per costruire alleanze forti contro le resistenze, conoscere i pregi e i limiti dei vari strumenti per poter tagliare. Chi si limita a imparare e ripetere il verbo tagliare e non cerca di coniugarlo nel modo giusto rischia di fare (e di far fare al SSN) la fine dei "pifferi di montagna che andarono per suonare ma furono suonati". Ciò vorrebbe dire imporre tagli nel breve periodo per dover subire aumenti di spesa nel medio e ancor più nel lungo periodo peggiorando nel contempo la qualità del SSN. 